Vol. 66, N. ${ }^{\circ}$ IO4 (noviembre 20I4), 6I-82

\title{
DIVERSIDAD Y CONVERGENCIAS EN LAS TEORÍAS DE LA DISTRIBUCIÓN: UN ESTUDIO RETROSPECTIVO
}

\author{
LUIS CÁRDENAS DEL REY \\ Universidad Complutense de Madrid
}

Recepción manuscrito: 1 de julio de 2014

Aceptación versión final: 30 de octubre de 2014

\begin{abstract}
RESUMEN En este trabajo se trata el origen y desarrollo posterior de la teoría de la distribución del ingreso. La principal cuestión que se trata es la posibilidad de establecer vínculos y, en última instancia, síntesis entre la distintas perspectivas. Para ello se seleccionan algunas de las formulaciones más significativas en la evolución del tratamiento teórico a los problemas de ciclo y distribución del ingreso, concretamente entre las décadas de los años cincuenta y setenta. Se va desde el modelo distributivo de Kaldor (1955), pasando por Pasinetti (1962), hasta Goodwin (1967), Kalecki (1971b) y Asimakopulos (1975).
\end{abstract}

PALABRAS CLAVE Enfoques heterodoxos, macroeconomía, teoría de la distribución del ingreso.

ABSTRACT This work is about the origin and subsequent development on the income distribution theory. The main point that is discussed here is the possibility to establish a link and, ultimately, a synthesis among these different perspectives. Hence, some of the most significant formulations on the evolution of the theoretical treatment of the problems about cycle and distribution of income have been selected, specifically between the decades of the 50s and 70s. This range starts with the Kaldor distribution model (1955), continues with Pasinetti (1962), Goodwin (1967), Kalecki (1971b), and ends with Asimakopulos (1975).

KEY WORDS Heterodox approaches, Macroeconomics, Income distribution theory.

JEL CODE B22, E11, E12, E32

\section{INTRODUCCIÓN}

Una de las principales preocupaciones de la Economía Política es la distribución funcional de la renta. Dentro de este marco de pensamiento se han desarrollado múltiples trabajos para abordar esta cuestión, especialmente desde que se han observado grandes cambios en la participación de la renta en la mayoría de países. La importancia que tiene la distribución de la renta sobre el ciclo económico permite hablar de una «teoría del ciclo endógeno» vinculada a la teoría de la demanda efectiva. Existe una creciente literatura que ha abordado estas cuestiones a nivel tanto teórico como empírico (para un mayor detalle de 
los desarrollos contemporáneos, véase Cárdenas, 2014), especialmente a partir del modelo seminal de Bhaduri y Marglin (1990). Caben destacar las contribuciones de Lavoie (1995, 2014), Blecker (2002), Palazuelos y Fernández (2007), o Palley (2014); muchos de estos trabajos reconocen la influencia de autores como Kaldor, Kalecki y Goodwin. Recientemente, Palley (2014) ha desarrollado un modelo que introduce los pagos realizados a los directivos basándose en la idea kaleckiana de poder de monopolio y conflicto distributivo de Goodwin. Ante estos avances, es conveniente reflexionar sobre las aportaciones de los economistas pioneros en este campo. En este trabajo se exponen cinco modelos formalmente matemáticos que forman parte de esta tradición de pensamiento, y que abordan los problemas de ciclo y distribución del ingreso desde perspectivas diferentes pero no incompatibles. Por tanto, la pregunta de investigación puede resumirse en: Partiendo de la existencia de una teoría de la distribución, ¿cuál ha sido su origen y su principal desarrollo?; ¿son sus planteamientos convergentes?

En el primer apartado se recoge el modelo distributivo de Kaldor (1955) que dará inicio a toda una línea de pensamiento, especialmente tras la amplicación de Pasinetti (1962) que permite relajar el supuesto de propensión a ahorrar nula por parte de los trabajadores; por otro en el que los ahorros de éstos se venden a los capitalistas por la tasa de ganancia ${ }^{1}$, este resultado es conocido como el Teorema de Pasinetti. Se realiza una sucinta exposición de dos modelos del economista polaco Michal Kalecki, donde se destaca el papel de la inversión en el desarrollo del ciclo económico y la (poco tratada) relación entre tendencia y ciclo. En este marco se encuentra el modelo de presa-predador propuesto por Richard Goodwin en 1967, donde dos poblaciones en un mismo territorio compiten y colaboran permanentemente, en este caso, capitalistas y trabajadores a través del salario real y la tasa de desempleo. Por último, se analiza el modelo propuesto por Asimakopulos, que mantiene todos los supuestos de las aportaciones tradicionales de Kalecki sobre distribución del ingreso y que se engloba de la primera generación de modelos neokaleckianos.

\section{LA PROPUESTA DE KALDOR}

En su artículo de 1955 titulado Alternative Theories of Distribution, Nicholas Kaldor revisa las distintas teorías de la distribución del ingreso (por lo que es un buen punto de partida para comenzar) que se habían propuesto hasta entonces; trata en primer lugar la obra de Ricardo, del que destaca el proceso de acumulación y la ley de rendimientos decrecientes que crean una tendencia al estado estacionario de la economía, donde finalmente cesa el proceso de acumulación. En segundo lugar, desarrolla la teoría de Marx, del que cuestiona esencialmente la Ley de Tendencia Decreciente de la Tasa de Ganancia (LTDTG) y la proletarización creciente; esta afirmación se debe a que considera por sus estudios empíricos que el salario real no había disminuido, de hecho, había aumentado en las dos fases del capitalismo, esencialmente el de tipo competitivo y el de tipo monopolista. En el apartado siguiente Kaldor expone el modelo neoclásico (en los que incluye a autores como Marshall y Wicksell) centrándose en la productividad marginal de los factores y el supuesto de una elasticidad de sustitución facturorial igual a la unidad, como los axiomas centrales de este enfoque teórico (Kaldor, 1995, p. 91): 
In fact, the whole approach which regards the share of wages and of profits in output as being determined by the marginal rate of substitution between Capital and Labour (...) is hardly acceptable to present-day economists. Its inadequacy becomes evident as soon as it is realized that the "marginal rate of substitution" between Capital and Labour -as distinct from the marginal rate of substitution between labour and land-can only be determined once the rate of profit and the rate of wages are already known.

A continuación, Kaldor resume la teoría de la competencia imperfecta, destacando la influencia del grado de monopolio siguiendo la obra de Kalecki, si bien posteriormente se muestra crítico con sus formulaciones posteriores al considerar que ha abandonado la influencia de la elasticidad de la demanda sobre el grado de monopolio volviéndose un enfoque tautológico ${ }^{2}$ (Kaldor, 1995, p. 92). En el siguiente apartado de este estudio se abordan en mayor profundidad las aportaciones de Michal Kalecki, pero Kaldor resume el efecto de la morfología del mercado sobre el grado de monopolio en la máxima: «Profit margins are what they are because the forces of competition preven it them from being higher than they are and are not powerful enough to make them lower than they are» (Kaldor, 1995, p. 93). Finalmente, propone un modelo que denomina «keynesiano» porque lo considera una evolución de los planteamientos de Keynes desde el Treatise. En primer lugar, plantea un modelo sin sector público y una economía cerrada, por tanto se comporta según las siguientes identidades:

$$
\begin{aligned}
& Y=W+P \\
& I=S \\
& S=S_{w}+S_{p} ; \quad S_{w}=s_{w} W \text { y } S_{p}=s_{p} P \\
& I=s_{w} W+s_{p} P \\
& I=s_{w} W+s_{w} P+s_{p} P-s_{w} P=s_{w} Y+\left(s_{p}-s_{w}\right) P
\end{aligned}
$$

Como es habitual, Y representa la producción, W los salarios, P los beneficios, I la inversión y $\mathrm{S}$ el ahorro. Al existir dos clases sociales, trabajadores y capitalistas, el ahorro debe distribuirse entre ambas, bajo el supuesto que los trabajadores no ahorran, si además denominamos $S_{w}, S_{p}$ al ahorro de trabajadores y capitalistas y $s_{w} y s_{p}$, a la propensión marginal. La expresión (2.d) indica cuál es la inversión si los trabajadores no obtienen parte de los beneficios; por el contrario (2.f) se les ha añadido en el reparto, para ello se suma y se resta el término $s_{w} P$ y, operando desde ese supuesto se obtiene (2.1) desde dónde empezamos el desarrollo:

$$
\begin{aligned}
& \frac{I}{Y}=s_{w}+\left(s_{p}-s_{w}\right) \frac{P}{Y} \\
& \frac{P}{Y}=\frac{1}{\left(s_{p}-s_{w}\right)} \frac{I}{Y}-\frac{s_{w}}{s_{p}-s_{w}} \\
& \frac{P}{K}=\frac{1}{\left(s_{p}-s_{w}\right)} \frac{I}{K}-\frac{s_{w}}{s_{p}-s_{w}} \frac{Y}{K}
\end{aligned}
$$


Operando la ecuación queda en función de la cuota de beneficio [2.2]. Kaldor denomina a como el «coeficiente de sensibilidad de la distribución del ingreso», que establece que cuanto mayor sea la diferencia entre la propensión a ahorrar de capitalistas y trabajadores, más crecerá la cuota de beneficio ante incrementos de la cuota de inversión. Además, es sencillo especificar la anterior relación en términos de la tasa de ganancia, sólo hay que despejar el output del lado izquierdo y dividir en ambos lados de la identidad por el stock de capital [2.2.1]. Si suponemos que la propensión a ahorrar de los perceptores de rentas del trabajo es nula entonces se obtiene la «Ecuación de Cambridge»:

$$
\begin{aligned}
& P=\frac{1}{s_{p}} I \\
& \frac{P}{Y}=\frac{1}{s_{p}} \frac{I}{Y} ; \text { donde } g_{n}=\frac{I}{Y}, g_{k}=\frac{I}{K} ; \text { entonces } \frac{P}{Y}=\frac{g_{n}}{s_{p}} ; \pi=\frac{g_{k}}{s_{p}}
\end{aligned}
$$

Que puede expresarse en términos de cuota de beneficios y cuota de inversión (gn), o también puede expresarse, una vez más, en términos de la tasa de ganancia $\pi$, de tal forma, que la expresión [2.4] defiende que la tasa de ganancia se compone de la tasa de acumulación, inversión sobre el capital (gk) y la propensión a ahorrar de los capitalistas. El resultado es bastante intuitivo, cuanto mayor sea la propensión a ahorrar de los capitalistas menos se incrementará la tasa de ganancia en cambios en la tasa de acumulación.

\section{EL TEOREMA DE PASINETTI}

El llamado Teorema de Pasinetti, tiene su origen en el artículo de 1962, Rate of profit and income distribution in relation to the rate of economic growth, escrito por Luigi L. Pasinetti. Donde se demuestra que las conclusiones obtenidas por Kaldor (1955) mediante su «propuesta keynesiana» de la widow's cruse, i. e., siguiendo la referencia bíblica utilizada por Keynes para describir una situación en el que el aumento del consumo de los empresarios aumenta el ahorro y los beneficios en su conjunto. En este modelo propuesto por Pasinetti siguen siendo válidas estas afirmaciones sin el supuesto de ahorro nulo por parte de los trabajadores. Por tanto, partiendo de los mismos supuestos que Kaldor, pero solucionando el supuesto, un tanto restrictivo, de este al no incluir a los trabajadores en los beneficios a repartir, se pueden especificar las ecuaciones de partida (ver Tabla 1). Operando de igual forma que en el trabajo de Kaldor, obtendremos las tasas de acumulación (I/Y), la participación de los beneficios del capital en la renta (Pc/Y), y la tasas de ganancia de los capitalistas como reordenación de la anterior.

$$
\begin{aligned}
& \frac{I}{Y}=s_{w}+\left(s_{c}-s_{w}\right) \frac{P_{c}}{Y} \\
& \frac{P_{c}}{Y}=\frac{1}{\left(s_{c}-s_{w}\right)} \frac{I}{Y}-\frac{s_{w}}{s_{c}-s_{w}} \\
& \frac{P_{c}}{K}=\frac{1}{\left(s_{c}-s_{w}\right)} \frac{I}{K}-\frac{s_{w}}{s_{c}-s_{w}} \frac{Y}{K}
\end{aligned}
$$


Tabla 1. Ecuaciones de partida de teorema de Pasinetti

\begin{tabular}{llll}
\hline$Y=W+P$ & {$[3 . \mathrm{a}]$} & $P=P_{w}+P_{c}$ & [3.d] \\
$I=S$ & {$[3 . \mathrm{b}]$} & $S_{w}=s_{w} W+S_{w} P_{w}$ & [3.e] \\
$S=S_{w}+S_{c}$ & {$[3 . \mathrm{c}]$} & $S_{c}=s_{c} P_{c}$ & [3.f] \\
\hline
\end{tabular}

El resultado es similar al que se llegó en Alternative Theories of Distribution, el problema es que el término del lado izquierdo de la identidad que refleja los beneficios de los capitalistas sobre el output no tiene significación económica, para resolverlo sumamos a ambos lados de la ecuación $P_{w} / K$ con lo que obtendremos de nuevo la tasa de ganancia $\mathrm{P} / \mathrm{K}$.

$\frac{P}{K}=\frac{1}{\left(s_{c}-s_{w}\right)} \frac{I}{K}-\frac{s_{w}}{s_{c}-s_{w}} \frac{Y}{K}+\frac{P_{w}}{K}$

Si definimos que los beneficios de los trabajadores es el capital que poseen por el precio que cobran por su préstamo, es decir, el tipo de interés, r, entonces se puede especificar: $P_{w}=r K_{w}$. Donde $\mathrm{Kw}$ es el stock de capital que pertenece a los trabajadores, que en este modelo a su vez se deriva de su capacidad de ahorro (Sw).

$$
\begin{aligned}
& \frac{P}{K}=\frac{1}{\left(s_{c}-s_{w}\right)} \frac{I}{K}-\frac{s_{w}}{s_{c}-s_{w}} \frac{Y}{K}+r \frac{K_{w}}{K} \\
& \frac{K_{w}}{K}=\frac{S_{w}}{S}=\frac{s_{w} s_{c}}{\left(s_{c}-s_{w}\right)} \frac{Y}{I}-\frac{s_{w}}{s_{c}-s_{w}} \\
& \frac{P}{K}=\frac{1}{\left(s_{c}-s_{w}\right)} \frac{I}{K}-\frac{s_{w}}{s_{c}-s_{w}} \frac{Y}{K}+r\left(\frac{s_{w} s_{c}}{\left(s_{c}-s_{w}\right)} \frac{Y}{I}-\frac{s_{w}}{\left(s_{c}-s_{w}\right)}\right)
\end{aligned}
$$

El ratio de capital que poseen los trabajadores $(\mathrm{Kw})$ y el total $(\mathrm{K})$ ha de ser necesariamente la ecuación [3.5] que resulta de operar con las definiciones iniciales. A partir de esa ecuación y sustituyendo el ratio entre capital que poseen los trabajadores y el total. Si suponemos que el tipo de interés es igual a la tasa de ganancia o en otras palabras que la rentabilidad obtenida en los mercados financieros iguala a la rentabilidad obtenida por operar en los mercados de bienes y servicios.

Operando se obtiene una vez más la Ecuación de Cambridge, donde la tasa de ganancia no depende ni del tipo de interés, ni de la renta, ni del ahorro de los trabajadores, pero esta vez sin el supuesto de ahorro nulo por parte de estos últimos: Expresado en términos de la cuota de beneficio o sólo de los beneficios el resultado es análogo:

$$
\frac{P}{K}=\frac{\left(s_{c}-s_{w}\right) I}{\left(s_{c} I-s_{w} s_{c} Y\right)} \frac{I-s_{w} s_{c} Y}{\left(s_{c}-s_{w}\right) K}=\frac{1}{s_{c}} \frac{I}{K} ; \text { por tanto } \frac{P}{Y}=\frac{1}{s_{c}} \frac{I}{Y} ;
$$

En el mismo artículo, Pasinetti expone una relación fundamental de los beneficios y el ahorro, partiendo de la igualdad de largo plazo entre los cocientes de ambos - beneficios y ahorros- por parte de las dos clases sociales se observa una razón por la cual la propensión a ahorrar de los trabajadores no juega ningún papel en la determinación de la tasa de ganancia. 
$\frac{P_{w}}{S_{w}}=\frac{P_{c}}{S_{c}} ; \frac{P_{w}}{S_{w}\left(W+P_{w}\right)}=\frac{P_{c}}{S_{s} P_{c}}=\frac{1}{S_{c}}$

$s_{c} P_{w}=s_{w}\left(W+P_{w}\right)$

$s_{w} W=s_{c} P_{w}-s_{w} P_{w}$

Se define que el ahorro total de los trabajadores va a ser igual a la cantidad que han ahorrado los capitalistas con los beneficios de los trabajadores. Además, su tasa de ganancia es indeterminada: Será una proporción de sus ahorros en el largo plazo, pero dependerá de la tasa de los capitalistas. Finalmente, como en el modelo de Kaldor tiene que cumplirse de nuevo la condición de estabilidad que establece que las propensiones al ahorro sean tales que la propensión a ahorrar de los trabajadores sea inferior a la tasa de acumulación y que la tasa de ahorro capitalista sea superior es decir: $s_{w}<I / Y ; s_{p}>I / Y$; en otras palabras, que se encuentre en el intervalo $s_{w}<I / Y<s_{p}$. Esto supone que ha de cumplirse el siguiente razonamiento lógico: Al concebir a los agentes económicos como clase, no puede producirse una situación en la que la clase trabajadora se convierta en capitalista y a la inversa, esto obviamente no es requisito a nivel individual, pero sí debe cumplirse esta estabilidad intergeneracional al tratarse como clase social en su conjunto. No es, por tanto, un mero formalismo matemático, sino que implica la coherencia de que los trabajadores ahorran una proporción menor de sus ingresos que los capitalistas y en consecuencia nunca podrán alcanzar su participación relativa en el stock de capital.

\section{EL PENSAMIENTO DE KALECKI}

El economista polaco Michal Kalecki realizó importantes aportaciones a la teoría del ciclo económico, por ello no puede considerarse un único modelo como el más representativo, de tal forma que fue incorporando nuevos elementos que dotaban de mayor realismo o mejoraban a los modelos anteriores, i. e., que eliminaban supuestos excesivamente restrictivos para aproximar los supuestos a priori a hechos observables en la realidad. Por tanto, es conveniente distinguir tres fases en la evolución de sus modelos. En el primero de ellos ya plantea la centralidad de la inversión como origen de las fluctuaciones, la inversión a su vez variaba en relación a la tasa de ganancia $(I / K=f(P / K, i)=F(P / K))$ manteniendo en ambos casos (con tipo de interés y sin él) una relación creciente.

En el siguiente modelo ${ }^{3}$, amplía los determinantes de la inversión, primero formula una ecuación que va a determinar la decisión de invertir (D) que Kalecki (1956) define como: «La cantidad de decisiones de invertir por unidad de tiempo [cursiva en el original]. Las decisiones de tal naturaleza efectuadas en un período dado, determinadas por ciertos factores que operan durante el período, son seguidas de cierto rezago por la inversión misma» (p. 67). Estas decisiones dependen positivamente del ahorro (S), de los beneficios obtenidos por los capitalistas (P) y negativamente del stock de capital existente (K), así como de una constante que refleja el movimiento determinista habitualmente positivo. En expresión matemática:

$D=a S+b \frac{\Delta P}{\Delta t}-c \frac{\Delta K}{\Delta t}+d ;$ 
Tabla 2. Ecuación del ciclo económico

\begin{tabular}{llll}
\hline Beneficios & & Producción \\
$I=S$ & {$[\mathrm{a}]$} & $V=\alpha Y+B ; \frac{V}{Y}=\alpha+\frac{B}{Y}$ & {$[\mathrm{e}]$} \\
$P=C+I$ & {$[\mathrm{~b}]$} & $Y_{t}=\frac{P_{t}+B^{\prime}}{1-\alpha^{\prime}}$ & {$[\mathrm{f}]$} \\
$C=A+q P$ & $O=Y+E$ & {$[\mathrm{~g}]$} \\
$P_{t}=\frac{I_{t-\omega}+A}{I-q}$ & {$[\mathrm{c}]$} & $O_{t}=\frac{P_{t}+B^{\prime}}{I-\alpha^{\prime}}+E$ & {$[\mathrm{~h}]$} \\
\hline
\end{tabular}

A partir de esta ecuación desagrega la inversión en factor fijo que obtiene sustituyendo la decisión de invertir por la inversión (F) en factor fijo: $F_{t+\theta}=a /(1+c) S+b^{\prime} \Delta P / \Delta t+d^{\prime}$ 'que a su vez se ha simplificado sustituyendo la variación del stock de capital por despejando $\Delta K / \Delta t=F_{t}-\delta$ y agrupando las variables mediante cambios de nomenclatura para obtener la función de inversión simplificada. Además se incluye la inversión en existencias $J_{t^{+} \tau}=e^{\prime} \Delta O / \Delta t$, que simplemente depende positivamente de la producción. La suma de ambos términos será la función de inversión. Puede observarse que ambos términos son dinámicos al incluir distintos períodos temporales pero la inversión no es contemporánea, sino que se determina para el período siguiente en función de su pasado, i. e., depende positivamente del nivel de ahorro presente $(\mathrm{S})$, de la tasa de variación de los beneficios $(\mathrm{P})$ y de la producción $(\mathrm{O})$, dando como resultado:

$$
I_{t+\tau}=\frac{a}{1+c} S+b^{\prime} \frac{\Delta P}{\Delta t}+e^{\prime} \frac{\Delta O}{\Delta t}+d^{\prime}
$$

Una vez expuesta su teoría de la inversión podemos desarrollar el capítulo 11 de Theory of Economic Dynamics (Kalecki, 1956), donde desarrolla la ecuación del ciclo y expone un modelo completo. Partiendo del supuesto de equilibrio en el comercio internacional y en el sector público, además se divide la sociedad en dos clases y se aplica el supuesto de que los trabajadores no ahorran; se definen las siguientes ecuaciones en la tabla 2.

Las ecuaciones de la izquierda son respectivamente, inversión total (I), ahorro total (S), beneficios netos $(\mathrm{P})$, consumo de los capitalistas (C), el componente autónomo del consumo de capitalistas (A) y un coeficiente de propensión a consumir los beneficios (q). La última ecuación de beneficios, se obtiene igualando las dos ecuaciones de consumo y despejando, además a la inversión ya se le ha descontado el efecto del sector público. Por otra parte, la columna de la derecha relaciona los sueldos y salarios (V) con los ingresos del sector privado (Y) más un coeficiente que indica la participación salarial $(\alpha)$, por su parte los ingresos del sector privado se determinan en función de las ganancias netas $(\mathrm{P})$, del componente autónomo del trabajo neto (B'), de la inversión (I) y de la participación salarial ( $(\alpha)$. El producto $(\mathrm{O})$ es la suma del ingreso bruto del sector privado (Y) más los impuestos (E), del cual se deriva la última ecuación de producto. La depreciación del stock de capital a lo largo del tiempo se define como $\delta$. La participación de la renta salarial $(\mathrm{w}=\alpha)$ es posible desarrollarla desde el lado del mark-up $(\mathrm{k}-1)$, 
donde la cuota salarial depende de los salarios brutos (W), y el coste de inputs (M), siendo la relación entre ambas, coste total de los materiales y los salarios (j); finalmente obtenemos:

$$
\begin{aligned}
& w=\frac{W}{W+(k-1)(W+M)} ; w=\frac{1}{1+(k-1)(1+j)} \\
& I_{t+\theta}=\frac{a}{1+c} I_{t}+\frac{b^{\prime}}{1-q} \frac{\Delta I_{t-\omega}}{\Delta t}+\frac{e^{\prime}}{(1-q)(1-\alpha)} \frac{\Delta I_{t-\omega}}{\Delta t}+d^{\prime} \\
& \left(I_{t+\theta^{-}}-\delta\right)=\frac{a}{1+c}\left(I_{t}-\delta\right)+\frac{1}{1-q}\left(b^{\prime}+\frac{e^{\prime}}{(1-\alpha)}\right) \frac{\Delta I_{t-\omega}}{\Delta t} \\
& i_{t+\theta}=\frac{a}{1+c} i_{t}+\mu \frac{\Delta i_{t-\omega}}{\Delta t}
\end{aligned}
$$

En la ecuación [4.4] se mantienen todas las relaciones previamente comentadas pero ahora están en una única ecuación, de tal forma que se observa un relación positiva respecto a los ahorros (a), negativa con el stock de capital (c), positiva respecto la variación de ganancias y producción. Este sistema puede expresarse de forma estática si se iguala de la inversión y la depreciación $\delta=\mathrm{I}$; y la tasa de variación es, obviamente, nula $(\Delta I / \Delta t)=0$. Aplicando esta característica a la relación [4.4] queda: $\delta=\frac{a}{1+c} \delta+d^{\prime}$. Si ahora se resta esta última ecuación a [4.4], entonces se consigue la evolución de la desviación de la inversión respecto a la depreciación [4.5]. y Definiendo $\left(I_{t}-\delta\right)=i_{t}, \frac{\Delta I_{t}}{\Delta t}=\frac{\Delta i_{t}}{\Delta t}, y \mu=\frac{1}{(1-q)}\left(b^{\prime}+\frac{e^{\prime}}{(1-\alpha)}\right)$, podemos simplificar términos. Siendo la expresión obtenida [4.6] la que va sustentar el análisis del ciclo económico y su mecanismo de funcionamiento, por ello se denomina «ecuación del ciclo económico».

El desarrollo es el siguiente: Considerando que el primer término $(a / 1+c)$, debe ser menor que 1 durante todo el período, se parte del supuesto origen en el cual la desviación de la inversión respecto a la depreciación (i) es cero, a partir de ese momento su tasa de variación es positiva, de tal forma que se incrementará hasta llegar a su límite superior, esto se debe a que se mantiene el término $(\mu)$ como positivo.

Pero este crecimiento se va reduciendo paulatinamente ya que el primer término (menor que uno) va «frenando» la inversión hasta que su variación es cero, este es el «tope» superior; por tanto, el crecimiento que se sustentaba en $\mu \Delta i_{t-\omega} / \Delta t$ desaparece porque hay un momento en que este miembro la variación deja de impulsar la fase alcista, por lo cual, empezará a caer la inversión, de nuevo, porque el primer término es menor que uno, esto implica que en el siguiente período la tasa de variación será negativa y la inversión caerá. Se inicia de esta forma una fase bajista de la fluctuación, ahora el «freno» va limitando la caída de la inversión hasta el tope inferior, que se producirá cuando la tasa de variación negativa desaparezca, una vez alcanzado el límite inferior, el proceso se reinicia con crecimiento hasta el siguiente límite, y así sucesivamente. Se considerarán los tramos de expansión y recesión cuando se situé en los puntos donde la inversión sea igual a la depreciación, es decir, cuando no exista diferencia entre ambas.

Lo más interesante de esta propuesta es que la «ecuación del ciclo económico», se compone de dos términos, uno dinámico que recoge la tasa de variación de la inversión y otro estático o de inercia que va a determinar la del período siguiente, como se ha comentado, actúa de tal forma que va reduciendo proporcionalmente la inversión hasta que finalmente la para. Es interesante desagregar este coeficiente en la propensión a reinvertir el ahorro (a) y el efecto 
del stock de capital (c), si la reinversión fuera lo suficientemente alta como para compensar el efecto perjudicial de la acumulación de capital, esto es si fuera igual a 1, entonces la inversión se mantendría prácticamente constante y tardaría mucho en reducirse ${ }^{4}$, especialmente si el stock de capital no reduce la determinación de la inversión.

Ambos conceptos están relacionados, cuando los capitalistas ven caer su tasa de ganancia desean reinvertir los ahorros en menor proporción, esto no solo intensifica (c) sino que también reduce (a), por ello, se produce antes la situación de límite superior, y a la inversa; pero no en igual proporción, pues se produce antes el deseo de dejar de invertir (al observarse un problema de realización) que la percepción de posible remuneración al haberse estancado el proceso de acumulación. El otro término, va a intensificar las variaciones de la inversión, cuanto mayor sea la propensión a consumir de capitalistas (q) y trabajadores más ampliará el efecto positivo de los beneficios ( $b^{\prime}$ ) y del crecimiento de la producción (e’). Por el contrario, cuando se produzcan las fases depresivas, y el deseo de invertir se reduzca, puede limitar su efecto.

Los problemas de este enfoque son dos, uno es que no mantiene elementos autónomos que permitan el crecimiento de base sobre el que se produce el ciclo, este es un hecho pretendido al eliminar las distintas constantes, en posteriores trabajos realizados por Kalecki se trata esta cuestión. El otro problema es su excesivo mecanicismo, i. e., que genera una dinámica automáticamente y siempre de magnitudes similares situación que obviamente no se produce en la actividad económica de una economía de mercado. Para solucionar este resultado se incorporan perturbaciones que siguen procesos puramente aleatorios, que por definición no son predecibles.

$$
i_{t+\theta}=\frac{a}{1+c} i_{t}+\mu \frac{\Delta i_{t-\omega}}{\Delta t}+\varepsilon
$$

Ahora, el ciclo es semiirregular, sus ondas no se determinan exclusivamente por el comportamiento de las variables y los coeficientes sino que mantiene un cierto grado de aleatoriedad que modifica las ondas por factores externos. La principal implicación es que no necesariamente la onda va a alcanzar los topes, si las perturbaciones las suavizan pueden darse los puntos de inflexión antes de llegar a los límites. Esto no elimina el efecto del ciclo endógeno, sino que lo complementa para dotarle de realismo en relación a las series observadas. En palabras de Kalecki (1956, p. 144):

Por lo tanto, aún con una atenuación relativamente fuerte, dichas perturbaciones generan ciclos bastante regulares. Este resultado tiene considerable importancia, pues indica que puede existir un ciclo semi-regular [sic] aún aún en el caso de que la "ecuación del ciclo económico" encierre bastante atenuación. En consecuencia, nos evita la necesidad de aceptar el ciclo explosivo como tipo general de fluctuación económica.

En resumen, en este modelo propuesto se relaciona la evolución de la inversión con el ciclo económico, mediante un procedimiento que de forma automática va generando fases regulares en función de la percepción de los agentes, en este caso los propietarios del capital, que van a crear las crisis al reducir el flujo de financiación; pero no sólo se centra en este hecho sino también la propensión al consumo de capitalistas y trabajadores (estos en cuota salarial pues consumen 
Figura 1. Diagrama de la ecuación del ciclo económico

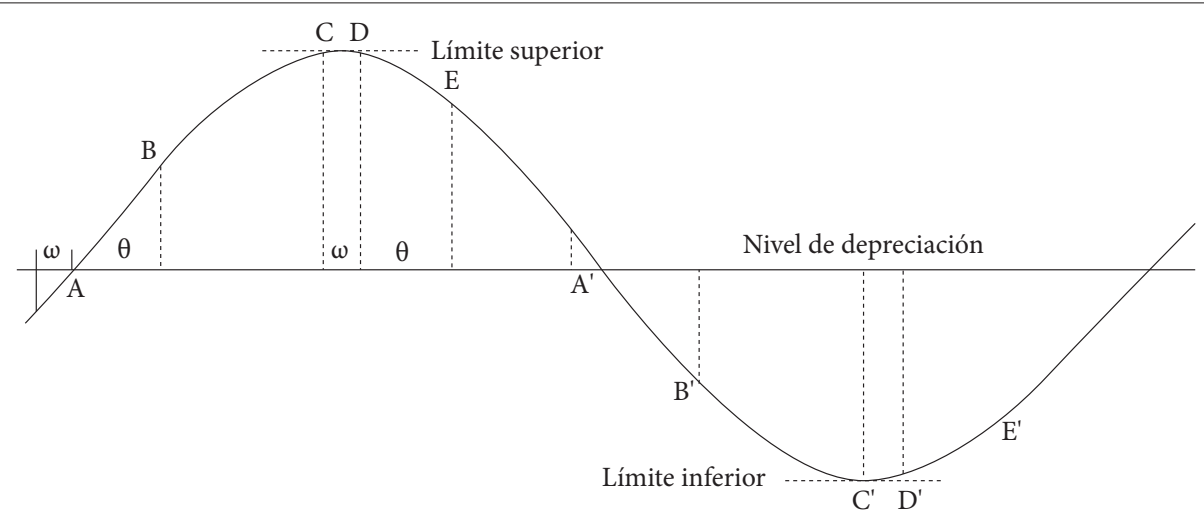

todo su salario) sirve de incentivo en las épocas de expansión y de freno en las de recesión, además incluye factores sociológicos en las decisiones de los agentes.

En 1968, Kalecki publica el que será su último trabajo sobre modelos de ciclo económico (su último trabajo se publicó póstumamente en 1971), Trends and business cycles reconsidered, en el que desarrolla un ciclo sobre una tendencia de largo plazo, pero define a la tendencia como un componente del ciclo que cambia lentamente, de tal forma, que no implica una situación de equilibrio, sino un factor menos volátil que el resto. Eliminando el supuesto de estacionaridad de la economía, plantea un modelo de "crecimiento cíclico». Al igual que en otros trabajos mantiene el axioma de la demanda efectiva y la centralidad de la inversión, a la que denomina la «central pièce de résistance of economics» (Kalecki, 1968, p. 263).

Primero, se formulan los supuestos habituales de economía cerrada y sin sector público donde los trabajadores consumen todos sus ingresos, no se diferencian los distintos momentos de consumo, se considera que todos los costes salariales están directamente implicados en la producción, es decir, son costes directos; por último, se elimina el componente de inventarios que se había expuesto previamente, dejando que las ganancias reflejen el factor de deseo de invertir por crecimiento de la producción. Quizás el aspecto más cuestionable sea el supuesto que la cuota de beneficios (q) sea un factor constante, esto implica que la distribución del ingreso no varía a lo largo del tiempo entre clases sociales, este hecho se debe a que todo el coste salarial es considerado directo a la producción, y en competencia monopolística, los precios se determinan mediante mark-up en función del grado de monopolio, si este es lo suficientemente alto, entonces transmitirán cualquier incremento salarial directamente en los precios; este no implica que haya utilización plena de factores o un equilibrio entre oferta y demanda a través de precios dados, sino que se debe a un factor institucional el grado de monopolio.

Se perfecciona la función de inversión considerara anteriormente, en este caso para analizar la decisión de invertir, se utilizan el beneficio esperado por utilización del equipo, la inversión necesaria para obtener esa ganancia, un coeficiente de deseo de inversión por ganancias, a ello hay que añadir la pérdida por mantener equipo viejo menos productivo, es decir, recoge la disminución de las ganancias por obsolescencia. Los determinantes de la inversión 
incluyen aspectos de toda índole, tal que: «To account for this additional stimulus to investment which is a direct outcome of [...] a slowly changing magnitude depending — similarly to the stable part of capitalists' consumption - on past economic, social and technological developments» (Kalecki, 1968, p. 269).

A partir de estos supuestos se obtiene una ecuación denominada «ecuación de la dinámica de inversión», definiendo una función estable en el tiempo o de tendencia que muestra tasas de variación reducidas, de ambas definiciones se deriva una función que genera automáticamente fluctuaciones cíclicas, muy similar a la «ecuación del ciclo económico». Pero ahora referenciada a un componente estable y no a un componente estacionario, como en el modelo detallado anteriormente, las fluctuaciones de la inversión determinan, como es habitual, la evolución de la producción (o ingreso nacional) y los beneficios.

El modelo concluye estudiando dos componentes que sólo influyen si consideramos la existencia de una tendencia, estos son la depreciación del stock de capital y el grado de utilización. Para el primero, utiliza la conocida ecuación de acumulación de capital se deduce que el límite de variación del stock de capital es también muy próximo a la variación otorgada al componente fijo. Sobre la utilización de la capacidad productiva, definiendo hK como la capacidad productiva, y tomando el componente tendencial del producto, el grado de utilización depende de la variación del componente fijo, del progreso técnico, del propio factor de obsolescencia y de la cuota de beneficios (q), que recordemos a su vez depende del grado de monopolio. En síntesis, del último modelo de Kalecki se deducen varios conceptos importantes, la relación existente entre el componente cíclico propiamente dicho y la tendencia, o variaciones de menor frecuencia, modificando el modelo que había desarrollado en Theory of Economic Dynamics, donde primero proponía el modelo de ciclo que hemos visto y le añadía una tendencia, que posteriormente despejaba y analizaba; ahora, el ciclo y la tendencia se forman desde el principio sin suponer nunca una economía de estado estacionario, se incluyen además una serie de temas que sólo es posible analizar si se amplía el espacio temporal más allá de las fluctuaciones, como son el progreso técnico, la depreciación del capital, no sólo en términos físicos sino también por obsolescencia consecuencia del propio progreso, la utilización de capital sin suponer que permanece constante indefinidamente, y la distribución del ingreso nacional donde juega un papel fundamental el grado de monopolio, que a su vez es consecuencia de diversos factores socioinstitucionales, como ya se ha expuesto previamente. En definitiva, Kalecki desarrolla un modelo que puede ser definido como de "dinámica cíclica», al incorporar elementos de fluctuaciones a corto plazo y de desarrollo tendencial de largo plazo.

No es difícil observar los principales rasgos de los keynesianos de Cambridge y del propio Keynes en el trabajo de Kalecki, mantiene la centralidad de la demanda agregada, especialmente mediante inversión, se recogen los efectos de los multiplicadores y el acelerador de la inversión, se construye sobre un mercado oligopólico sin necesidad de recurrir a la competencia perfecta como había tenido que hacer el propio Keynes, tampoco se cumple la ley de Say, se recogen factores sociales y de distribución del ingreso, se le otorga un papel a las expectativas en relación a la existencia de capital, beneficios y producción. De igual forma se distingue una síntesis de la obra de Marx en el papel de la acumulación y la tasa de ganancia con la demanda agregada y la competencia monopolística; y todo ello de forma dinámica, 
sin necesidad de recurrir a planteamientos de equilibrio parcial o general. Si además se considera que cuando formuló sus modelos se encontraba en auge la economía cuantitativa y formalmente matemática frente a la anterior modelización discursiva que había sido dominante entre los economistas decimonónicos, siendo el mejor ejemplo la General Theory de Keynes donde sólo se utilizan ecuaciones simples y un único gráfico.

Por el contrario, Kalecki siempre consideró los modelos formalmente matemáticos como herramientas prácticas que debían ser contrastadas permanente con la realidad, mediante ejemplificaciones estadísticas y la utilización con cierta frecuencia de las herramientas econométricas, e incluso desarrollando simulaciones incluyendo las perturbaciones aleatorias (en las que obtuvo buenos resultados) (Kalecki, 1956).

Posiblemente sea debido a su formación como ingeniero, pero también a la influencia de autores como Rosa Luxemburgo con la que compartía la importancia del papel de la acumulación, el sector exterior y el gasto del gobierno, aunque también existen planteamientos contradictorios entre ambos (López y Assous, p. 195). Con este bagaje, llegó a considerar que «la econometría y el materialismo histórico no eran irreconciliables» (Feiwel, 1981, p. 103). Esta idea se basa en sostener que el objeto de ambos enfoques es complementario, por un lado al estar el análisis econométrico basado en la utilización de variables en las que las relaciones sociales de producción que las desarrollaron se encuentran dadas, en términos econométricos para un período muestral establecido.

Y por otro lado, al estudiar el materialismo histórico la evolución de la estructura y la superestructura de la sociedad y los procesos de retroalimentación que se encuentran intrínsecamente asociados a la misma, es decir, el proceso de transformación dialéctico. Este punto de vista se encuentra claramente reflejado en varios trabajos, especialmente cuando afirma que una situación de pleno será insostenible durante mucho tiempo debido a la oposición de los empresarios, dado que «Their class instinct tells them that lasting full employment is unsound from their point of view and that unemployment is an integral part of the "normal" capitalist system» (Kalecki, 1943, p. 326). Por todo ello, es sencillo entender porque muchos autores de diversos ámbitos, incluida toda una corriente de la escuela poskeynesiana, se reconocen deudores de su pensamiento. Por tanto, los fundamentos teóricos del pensamiento kaleckiano analizados en este estudio se pueden resumir en las siguientes propiedades:

- La decisión de invertir queda en manos de los capitalistas (partiendo del supuesto de que la sociedad se divide en dos clases, por un lado propietarios y gestores del capital, y por otro lado los trabajadores), siendo esta la variable central para comprender la acumulación de capital. Los capitalistas pueden decidir la cantidad a invertir pero no cuál va ser el beneficio, a su vez las decisiones de inversión dependen de la rentabilidad esperada y un conjunto de factores sociales, tecnológicos y de inercia.

- El proceso de determinación de precios se desarrolla en mercados con una organización competitiva monopolística u oligopolística, con existencia de poder de mercado. Para representar la fijación de precios se utilizan métodos de mark-up y cost-plus, i. e., se aplica el margen que puede imponer la empresa sobre el coste unitario directo.

- En las economías de mercado (laissez faire) la oferta de bienes y servicios terminados es 
Tabla 3. Modelo presa-predador, supuestos de partida

\begin{tabular}{llll}
\hline$a=a_{0} e^{\alpha t} ; \alpha>0$ & {$[5 . \mathrm{a}]$} & $u=\frac{w l}{q}=\frac{w}{a}$ & {$[5 . \mathrm{e}]$} \\
$n=n_{0} e^{\beta t} ; \beta>0$ & {$[5 . \mathrm{b}]$} & $v=\frac{l}{n}$ & {$[5 . \mathrm{f}]$} \\
$\sigma=\frac{k}{q}$ & {$[5 . \mathrm{c}]$} & $\dot{k}=(1-u) q$ & {$[5 . \mathrm{g}]$} \\
$l=\frac{q}{a}$ & {$[5 . \mathrm{d}]$} & $\frac{\dot{w}}{w}=-\gamma+p v, \operatorname{con} \gamma y p>0$ & {$[5 . \mathrm{h}]$} \\
\hline
\end{tabular}

Fuente: Kalecki (1956, p. 125).

elástica a corto plazo, de ello se deriva que a corto plazo una aumento de demanda se compensa un mayor grado de utilización, y no necesariamente con situaciones inflacionistas, con la única posible excepción de la fase más alcista del ciclo económico. De igual forma, no existe una situación ad hoc de pleno empleo, sino que generalmente este se encuentra subutilizado como consecuencia de la debilidad de la demanda agregada, del desempleo tecnológico, o por aspectos de su carácter de medida disciplinaria.

- La dinámica macroeconómica combina elementos cíclicos y tendenciales, esto implica que las fluctuaciones de corto plazo o ciclos cortos se desarrollan sobre un componente que avanza de forma más lenta al que se define como tendencia. Que el ciclo evolucione sobre un componente no estacionario es sin duda una aproximación muy original a la dinámica macroeconómica.

\section{EL MODELO PRESA-PREDADOR DE GOODWIN}

Richard M. Goodwin publicó en 1967 un modelo que integraba la concepción marxista del ejército industrial de reserva con las oscilaciones cíclicas y el desarrollo de largo plazo, el título de A Growth Cycle es acertado pues combina los elementos de fluctuaciones con conceptos de largo plazo, progreso técnico y acumulación de capital. Es conveniente señalar las diferencias con el pensamiento de Kalecki:

- Goodwin otorga la centralidad del modelo al conflicto distributivo directamente, relacionando desempleo y cuota salarial de la distribución, bajo el supuesto implícito de un mercado de trabajo que no se vacía necesariamente, $i$. e., un típico mercado laboral keynesiano.

- La inversión se realiza de forma inmediata, como veremos, esto implica que el crecimiento de la producción es directamente la tasa de ganancia, se han eliminado todos los supuestos kaleckianos de decisiones de inversión, retardos en las variables, incentivos y deseos de financiarse, en el planteamiento de Goodwin, se pierde la descomposición entre un componente estático de decisión de invertir y otro de acelerador de la propia inversión.

- Tampoco se analizan los efectos en tendencia y oscilación, de tal forma que se aplica directamente sin descomponer sus efectos. Además, el ciclo se reproduce sobre el mismo punto 
Figura 2. Diagrama de presa-predador

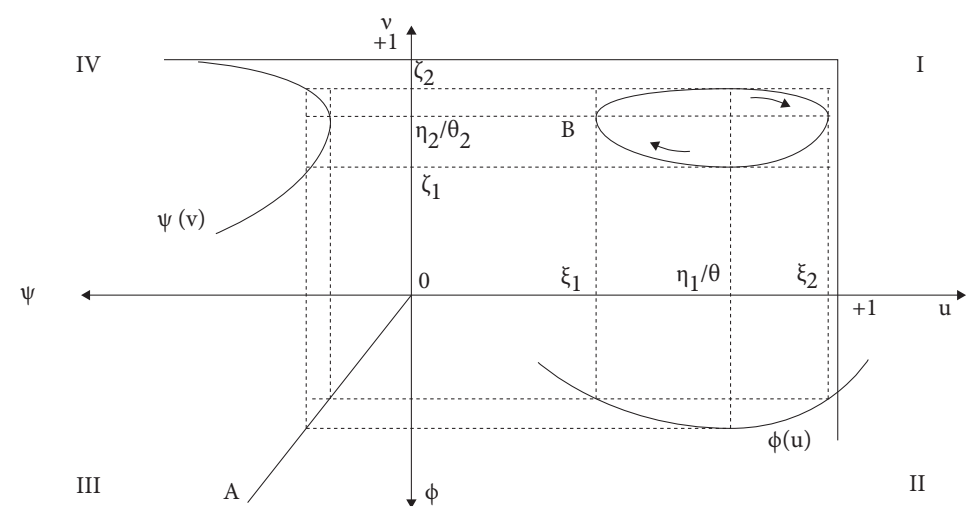

dando cierto mecanicismo al proceso, igual que sucedía en algunos de los modelos expuestos previamente en este trabajo.

La principal similitud es el papel de la acumulación de capital, no en vano ambos pueden ser considerados marxistas en un sentido laxo (aunque quizás Goodwin se adapte mejor a esta etiqueta), ambos mantuvieron estancias en la Universidad de Cambridge y ambos pueden ser considerados dentro de la primera generación de la escuela poskeynesiana, también conocida como Escuela de Cambridge (UK), esto hace que sus diferencias sean menores comparadas con sus puntos en común.

Además, realiza otra serie de supuestos, los de tipo teórico: existe un progreso técnico exógeno, un crecimiento continuo de la mano de obra, los salarios se consumen en su totalidad y los beneficios se ahorran e invierten, como en los modelos típicamente kaleckianos. Los supuestos de tipo empírico: un ratio constante entre capital-producto y un salario real que aumenta cuanto mayor es el empleo.

Su comportamiento consisten en productividad (a) constante, siendo a la tasa de crecimiento de la productividad, a su vez hay crecimiento poblacional constante (n) a una tasa la relación capital-producto $(\sigma)$, es constante, siendo respectivamente $\mathrm{k} \mathrm{y} \mathrm{q,} \mathrm{el} \mathrm{capital} \mathrm{y} \mathrm{la} \mathrm{pro-}$ ducción, la demanda de trabajo (l) depende positivamente de la producción y negativamente de la productividad. Como la cuota salarial (u) depende de la cantidad de trabajo, es fácil deducir que será creciente con la renta salarial y decreciente con la productividad. Por su parte, la tasa de ocupación (v) es lógicamente los trabajadores contratados sobre la población total; para obtener la inversión tenemos que considerar que sólo ahorran los capitalistas e invierten todo su ahorro, además hay una igualdad contemporánea entre ahorro, inversión y variación del stock capital, $(S=I=\dot{k}$ ), esto implica que la evolución del capital será exactamente la participación de los capitalistas en el producto nacional.

Por último, la tasa de variación de los salarios se calcula mediante una curva de Phillips, $\dot{w} / w=f(v), f^{\prime}(v)>0$, que indica la relación creciente entre el crecimiento de los 
Tabla 4. Nomenclatura del modelo kaleckiano de Asimakopulus

\begin{tabular}{|c|c|c|c|}
\hline Salario nominal unitario & $\mathrm{w}$ & Masa salarial & $\mathrm{W}$ \\
\hline Producto medio por unidad & $\mathrm{a}$ & Valor total de & \\
\hline de mano de obra directa & & los beneficios & $\Pi$ \\
\hline $\begin{array}{l}\text { Empleo de la mano } \\
\text { de obra directa }\end{array}$ & $L_{1}$ & $\begin{array}{l}\text { Valor total del } \\
\text { consumo }\end{array}$ & $\mathrm{C}$ \\
\hline Empleo máximo posible & & Elemento constante del consumo & \\
\hline con la capacidad instalada & $L_{1}$ & de los capitalistas & A \\
\hline Empleo de la mano de obra & & Pequeña fracción que expresa & \\
\hline no relacionada & & la dependencia de una parte & \\
\hline con la producción & & del consumo de los capitalistas & $\lambda$ \\
\hline sobre los beneficios actuales & $L_{0}$ & & \\
\hline Total de empleados & $L$ & Valor total del ahorro & S \\
\hline Precios de la producción & $\mathrm{p}$ & Valor total de la inversión & I \\
\hline $\begin{array}{l}\text { Margen sobre coste } \\
\text { unitario directo }\end{array}$ & $\mu$ & $\begin{array}{l}\text { Inversión bruta en } \\
\text { términos reales }\end{array}$ & I \\
\hline Valor de la producción bruta & $\mathrm{Y}$ & & \\
\hline
\end{tabular}

salarios y el nivel de ocupación. De estos supuestos es sencillo deducir algunas relaciones, la tasa de ganancia, como los beneficios son exactamente la variación del capital, entonces $r=\dot{k} / k=(1-u) q / k=(1-u) / \sigma$; que mantiene la habitual relación creciente respecto beneficios y negativa respecto stock de capital; además como la relación capital-producto es estable, cualquier incremento de capital aumentará la producción en la misma cantidad proporcional, por ello la tasa de ganancia marca el crecimiento del output $r=k / k=\dot{q} / q$. Con el sistema de ecuaciones propuesto se obtienen las siguientes relaciones fundamentales ${ }^{5}$ :

$$
\begin{aligned}
& \dot{u}=[-(\alpha+\gamma)+p v] u \\
& \dot{v}=\left(\frac{1-u}{\sigma}-(\alpha+\beta)\right) v
\end{aligned}
$$

Este sistema de ecuaciones nos indica que la variación temporal de la renta salarial depende negativamente del progreso técnico y de la constante asignada y, positivamente de la ocupación; mientras que la variación en el nivel de empleo depende de la producción, que como hemos visto es la tasa de ganancia, y negativamente del crecimiento de la población y del progreso técnico. Existe una fuerte similitud con las conocidas ecuaciones de Lotka-Volterra, propuestas por Alfred J. Lotka y Vito Volterra consisten en dos ecuaciones diferenciales no lineales que han sido utilizadas en el campo de la biología matemática para estudiar la dinámica entre dos poblaciones (o más) que se comportan tanto de forma colaborativa como hostil al convivir en un mismo territorio. La existencia de conflicto entre los grupos es la razón por la que estas ecuaciones son popularmente conocidas como Predator-Prey model o modelo de presa-predador.

Goodwin consideraba que era una buena comparación del sistema capitalista según la concepción de la tradición marxista, aunque como en este caso son los trabajadores los 
depredadores y los capitalistas la presa, es una metáfora del proceso dialéctico de la historia. Finalmente, derivando sobre los nuevos términos conseguimos ${ }^{6}$ :

$$
\begin{aligned}
& \frac{\partial \Phi}{\partial u}=\left(\frac{-\theta_{1}+\eta_{1}}{u}\right) \Phi \\
& \frac{\partial \Psi}{\partial v}=\left(\frac{-\theta_{2}+\eta_{2}}{v}\right) \Psi
\end{aligned}
$$

Para explicar $\Phi(u)=H \Psi(v)$, es útil desarrollar un diagrama con cuatro cuadrantes donde todas las relaciones se consideran en términos positivos, partiendo del origen se traza la línea A que tiene como pendiente la constante $(\mathrm{H})$, si sitúa en el cuadrante inferior izquierdo (III). A partir de esta línea se pueden conseguir las relaciones en derivadas (3) y (4), la primera en el segundo cuadrante (II) y la (4) en el cuadrante IV. La relación entonces es inmediata, existe una curva positiva cerrada sobre el punto B, como puede observarse en el primer cuadrante (I); es en este punto donde se produce el comportamiento cíclico, su localización va a depender de la línea de origen $\mathrm{A}$, que hemos elaborado con la pendiente de $\mathrm{H}$, así cambios en la pendiente modificaran la localización de la curva cerrada. La variación se produce por cambios en la composición de los términos, cuando la tasa de ganancia aumenta, la producción creciente requiere mayor mano de obra, por lo que el desempleo disminuye, esta situación provoca un aumento de los salarios reales que a su vez disminuye la tasa de ganancia, aumenta el desempleo y se reinicia el ciclo. Harvie (2000) realiza un proceso de linearización para conseguir la ecuación que representa el tiempo que dura el ciclo, así $T=2 \pi /((\alpha+\gamma)(1 / \sigma-(\alpha+\beta)))^{1} / 2$; pero nada indica la amplitud de la onda, por lo que en última instancia es un supuesto realizado ad hoc (ver Figura 2).

Se pueden obtener las ecuaciones de estado estacionario, bajo el supuesto de variaciones nulas, con lo que el sistema (5.1)-(5.2), pasa a ser un modelo con dos ecuaciones y dos incógnitas, de tal forma que se obtiene fácilmente:

$u^{*}=1-(\alpha+\beta) \sigma$

$v^{*}=(\alpha+\gamma) / p$

Las conclusiones que se pueden obtener del modelo de Goodwin son varias, la existencia del ejército industrial de reserva implica reacciones en los salarios reales, si es reducido los salarios serán altos y a la inversa; que el crecimiento de la economía sea directamente la tasa de ganancia es un instrumento que permite modelizar de forma sencilla el papel de la inversión, aunque en realidad es la tasa de ganancia (los capitalistas) la presa que es perseguida por el depredador (los trabajadores). Esta situación se produce por dos motivos, el suponer una proporción constante entre capital-producto y la acumulación de capital excesiva cuando la producción aumenta, ambas características son las causas de que la reproducción del capital cree su propia fase descendente del ciclo. Otra nota interesante es entender el progreso técnico en función del paro tecnológico, hemos visto que en este modelo la evolución de la técnica y el crecimiento de la población reducían el salario al aumentar el ejército industrial de reserva, por ello, entiende implícitamente que el capital recibe los beneficios del progreso técnico. 


\section{EL MODELO KALECKIANO DE ASIMAKOPULOS}

El economista canadiense Athanasios Asimakopulos sintetizó las teorías kaleckianas de la distribución del ingreso y la lucha de clases en A Kaleckian theory of income distribution, en Canadian Journal of Economics, profundizando en los conceptos que han sido tratados de forma más secundaria, además, este modelo es interesante por suponer un paso intermedio entre los primeros planteamientos de Kalecki y la actual corriente kaleckiana, i. e., un "puente» para transitar entre ambas formalizaciones. El artículo comienza exponiendo la teoría del mark-up, podemos afirmar que los precios se forman según un análisis del coste unitario de producción, así tomarían la forma $p=m u+n p$, donde $\mathrm{p}$ es el precio $\mathrm{y} \mathrm{u}$ el coste directo por unidad, el factor $n$, va a suponer la ponderación del precio medio de la industria $(p)$ si restamos y dividimos en ambos lados de la ecuación el coste (u) obtenemos el margen aplicado: $(p-u) / u=m-1+(\bar{n} p) / u$, presenta dos características fundamentales, la dependencia de otros precios de la industria y, que el grado de monopolio puede incrementarse pero nada indica que lo tenga que hacer obligatoriamente.

Mientras que las variaciones de corto plazo de los precios están determinadas por la evolución del mark-up, la evolución de largo plazo está determinada por la demanda (Kalecki, 1956, p. 11), como consecuencia que las economías de mercado operar por debajo de su capacidad la oferta de bienes es suficientemente elástica para cubrir la demanda a corto plazo, por el contrario la demanda de bienes intermedios e inputs primarios o materias primas responden con mayor lentitud. En este punto conviene recordar los factores que recoge Kalecki (1956, p. 1719) para explicar la evolución del grado de monopolio se encuentran: (1) la concentración de la industria en empresas oligopólicas con fuerte poder de mercado; (2) la utilización de la publicidad y campañas de venta para reemplazar la competencia mediante los precios en una basada en la comercialización de productos; (3) las variaciones de los gastos generales en su relación con los costes primarios; y (4) la fuerza que ejercen los sindicatos obreros evitará en cierta medida que se eleve el grado de monopolio. Se observan varios de estos elementos en el modelo propuesto por Asimakopulos, que incluye mark-ups diseñados para cubrir a lo largo del tiempo los costes generales (overhead o no directamente relacionados con la producción) y los beneficios, por tanto son dependientes de la utilización de la capacidad productiva y de la tasa de rendimientos esperada puesto que el margen debe procurar garantizarla. Por brevedad, se resume la nomenclatura utilizada en el modelo en la tabla 4.

En esta línea, los precios en términos agregados va a ser función creciente del margen, del salario y decreciente de la productividad laboral, así $p=(1+\mu)(w / a)$, por su parte la pro-

ducción desde el lado de la oferta será $Y=$ pa $L_{1}$; con $L_{1} \quad{ }^{-} L_{1}$ y desde el lado de las rentas $Y=W+\Pi=w L+\Pi$; hay que considerar que el número total de trabajadores se descompone en este modelo entre trabajadores directamente relacionados con la producción y aquellos que se consideran generales, así $L=L_{0}+L_{1}$. El consumo y el ahorro mantienen los supuestos habituales de que los trabajadores no ahorran pero los capitalistas si consumen y, existe igualdad entre ahorro e inversión, por lo tanto, $C=W+\lambda \Pi+p A \mathrm{y}, S=(1-\lambda) \Pi-p A$.

Por su parte la inversión $(I=\bar{p} I)$, va estar determinada en un primer momento por las decisiones de invertir, es decir, va a ser una variable exógena en el corto plazo, y va a estar limitada por dos situaciones, por una parte el límite de la capacidad instalada y la fuerza de trabajo 
disponible y, la otra, el control y la estabilidad de precios, que pueden activar mecanismos de desincentivar la inversión. A partir de la ecuación de los ahorros y la inversión, bajo la condición $I=S$, llegamos a la ecuación de los beneficios $\Pi=p(I-A) /(1-\lambda)$, desde esta ecuación se pueden realizar dos operaciones, obtener los beneficios en términos reales dividiendo ambos lados por los precios, o se puede sustituir la ecuación de precios, previamente expuesta se obtiene (6.1), sustituyendo en la ecuación de la producción y operando se obtiene las ecuaciones de número de ocupados, pudiendo expresarse de dos formas (6.2) y (6.3).

$\Pi=\frac{(1+\mu) w}{a} \frac{(I+A)}{(1-\lambda)}$

$L_{1}=\left(\frac{1}{\mu}\right)\left(L_{0}+\frac{(1+\mu)(I+A)}{a(1-\lambda)}\right)$

$L_{1}=\left(\frac{1}{\mu}\right)\left(L_{0}+\frac{p(I+A)}{w(1-\lambda)}\right)$

En la ecuación (6.3) se ha multiplicado y divido el último término por el salario unitario, de tal forma que vuelven a aparecer los precios en la ecuación.

$$
\frac{\Pi}{Y}=\frac{(\mu(I+A))}{\left(a(1-\lambda) L_{0}+(1+\mu)(I+A)\right)}
$$

Este modelo presenta unas características bastante interesantes, la masa de beneficios en términos reales no depende de los precios, esto implica que el mercado de competencia imperfecta va a transmitir siempre cualquier proceso inflacionario, manteniéndose en términos de la inversión y ampliándose su efecto cuanto mayor sea el consumo de los capitalistas (1- $\lambda$ ). Por el contrario, la cuota de ganancia (6.4) si depende positivamente del margen que aplican las empresas, pero también de otros factores como el consumo de los capitalistas, la productividad, el multiplicador y el trabajo no aplicado a la inversión; este resultado no es inamovible sino que varía, inter alia, en función de las circunstancias institucionales, como el poder de negociación colectiva o el estado de la inflación. Los supuestos aplicados para obtener estos resultados son muy próximos a los utilizados por Kalecki, así según Asimakopulos (1975, p. 327):

This Kaleckian theory of distribution combines two of Kalecki's important contributions: his recognition of the role of the degree of monopoly in the setting of mark-ups, and his demostrantion of the role of capitalist's expenditures in determining profits and the level of employment».

La diferencia con el método utilizado en Class Struggle and the Distribution of National Income, es que no es necesario recurrir a modelos de tres departamentos como en los análisis de la tradición marxista, sino que es perfectamente válido en un modelo unisectorial en el que se logran conclusiones en la misma dirección. Finalmente, se distingue otro postulado en el modelo que va a ser posteriormente explotado por múltiples autores (Bhaduri y Marglin, 1990), y es la posibilidad que el aumento de la participación de la renta de los trabajadores en el ingreso nacional incremente ante la plena utilización (o la proximidad a la misma) y la mayor capacidad de negociación que lleva asociada ${ }^{7}$. 


\section{CONCLUSIONES}

La teoría de la distribución del ingreso ha sido desarrollada en la tradición de la Economía Política por distintas escuelas, keynesianos y marxistas fundamentalmente, el axioma esencial compartido por todos los enfoques es la existencia de distintas clases sociales - en una economía capitalista resumidas en capital y trabajo- que se encuentran en conflicto por los rendimientos de la producción. A partir de este punto se desarrollan elementos analíticos sobre la influencia en el ciclo económico en distintas direcciones. El primer tipo de modelos, de Kaldor y Pasinetti, puede resumirse en la vieja máxima de «los capitalistas ganan lo que gastan y los trabajadores gastan lo que ganan,» (Kaldor, 1955, p. 96); es decir, la inversión va a determinar los beneficios a lo largo del ciclo.

En segundo lugar, el planteamiento de Kalecki de la «ecuación del ciclo económico», compuesto de un parte de dinámica de la inversión y otro elemento estático o de inercia que va a actuar de freno, es bastante completo (por ello sus modelos herederos mantendrán la denominación de kaleckianos, cf. Lavoie, 1995). Mantiene la centralidad de la demanda agregada, el principio de demanda efectiva, especialmente en inversión, se recogen los efectos de los multiplicadores y el acelerador, el modelo se construye sobre un mercado oligopólico donde no se cumple la ley de Say por la existencia de capacidad productiva infrautilizada. Asimismo, se le otorga un papel a factores sociológicos como las expectativas en relación a la existencia de capital, beneficios y producción, o el grado de monopolio, que a su vez depende de, inter alia, la fortaleza en la capacidad de negociación de los trabajadores. En esta línea el modelo de Asimokopulos permite entender el proceso inflacionario como un conflicto distributivo, pero incluyendo en los determinantes de los beneficios del margen (mark-up) que aplican las empresas, pero también de otros factores como el consumo de los capitalistas, la productividad, el multiplicador y el trabajo no aplicado a la inversión; este resultado no es inamovible sino que varía en función de las circunstancias institucionales, como el poder de negociación colectiva o el estado de la inflación.

Respecto a las principales ideas de Goodwin, desde esta perspectiva se enfatiza la existencia del ejército industrial de reserva y su relación con los salarios reales. La evolución cíclica establece que ante una reducción del ejército industrial se produce un aumento en los salarios reales, debido al mayor poder de negociación derivado de la escasez relativa del factor trabajo. Cuando esta situación provoca una caída de la rentabilidad se produce el mecanismo en sentido inverso, la caída de la actividad aumenta el desempleo y reduce los salarios reales. Además la incorporación del progreso técnico y, como consecuencia del paro tecnológico, reducen el salario real, de ello se deduce que el capital recibe los beneficios de dicho progreso técnico.

La respuesta a la posibilidad de una síntesis es positiva. Considera que en torno al núcleo formado por la teoría macroeconómica kaleckiana es posible desarrollar las principales aportaciones de las otras dos perspectivas recogidas. Para ello deben incorporar los siguientes elementos:

- De los trabajos de Kaldor y Pasinetti se incorpora una función de ahorro basada en la ecuación de Cambridge, con su corolario de que afirma que un incremento de la propensión a ahorrar por parte de los capitalistas deprime la economía y los beneficios. Asimismo, el Teorema de Pasinetti está incorporado de facto, en la mayoría de modelos kaleckianos modernos, puesto que los resultados son idénticos a considerar una propensión al ahorro 
positiva de los trabajadores (Blecker, 2002) siempre que se cumpla la condición de que la propensión a ahorrar de los trabajadores no supere la propensión de ahorro de los empresarios.

- Conflicto entre las clases sociales. Si bien el concepto de lucha de clases está presente en la obra de Kalecki, tiene un carácter secundario en comparación con los planteamientos de Goodwin, donde la dinámica está determinada por el tamaño del ejército industrial de reserva, en función del cual responden todas las variables. La otra idea que podemos extraer de la formulación de Goodwin es que el progreso técnico beneficia (en mayor medida a los empresarios).

- No cabe duda alguna de la importancia otorgada por Kalecki a los determinantes de la función de inversión, entre los cuales destaca la rentabilidad esperada de la inversión asociada a su vez al estado de la demanda efectiva, pero también otros de muy distinta índole como los factores socioinstitucionales entre los que se encuentran la capacidad de negociación de los trabajadores y la percepción que tengan de la misma los empresarios; los factores tecnológicos como la obsolescencia y el ratio de reemplazamiento de los equipos industriales, junto con factores que recogen el path-dependence en el proceso de acumulación.

- La formación de precios se basa en el concepto de grado de monopolio y aplicación de márgenes. La microfundamentación monopolística es una característica esencial para abordar como se determinan los beneficios en una economía capitalista, la evolución de la morfología del mercado como resultado de la acumulación y centralización del capitalson centrales a la hora de establecer los beneficios y la rentabilidad.

- Una posilidad es abordar la segmentación del mercado laboral, a partir del modelo de Asimakopulos es fácilmente abordable si en vez de considerar como trabajo general y concreto se reinterpreta como trabajadores pertenecientes al mercado primario y como trabajadores pertenecientes al mercado secundario (cf. Palley, 2014).

En resumen, es posible afirmar que la teoría de la distribución y el ciclo económico tiene un origen diverso, con perspectivas en ocasiones fuertemente divergentes, pero a lo largo de los desarrollos producidos en el siglo XX, las distintas posturas han convergido hacía un único cuerpo teórico que incorpora elementos de todas las perspectivas. La clave de bóveda que une todas estas visiones posiblemente se encuentre en torno a las aportaciones centrales del pensamiento de Kalecki.

\section{NOTAS}

1 El término tasa se utiliza exclusivamente para reflejar la relación entre dos variables que no forman parte del mismo conjunto, por ejemplo, la tasa de ganancia refleja la relación entre beneficios y capital. Por el contrario, el término cuota se utiliza exclusivamente para referirse a la relación entre variables que participan en otra variable, por ejemplo, la cuota de beneficios mide la relación entre los beneficios y la renta total del país, dado que la renta total es la suma de los salarios y los beneficios pertenecen al mismo conjunto de variables del cual se derivan.

2 Kalecki (1968, p. 265) responde a esta afirmación: «If the price is not determined by the equilibrium of supply at full utilisation of equipment, on the one hand, and demand, on the other, the prices are fixed by the firms on the basis of the average prime costs and the average price of the product group in question. The "pricing formulae" of firms determine, as I showed, the ratio of aggregate proceeds to aggregate prime costs. It is the set of the parameters of these formulae that constitutes the "degree of monopoly"». 
3 Para elaborar esta sección se ha utilizado tanto las obras de Kalecki (1977) como de Kalecki (1956) indistintamente; en estos trabajos se recogen en profundidad los argumentos y desarrollos que se han obviado en este estudio por brevedad.

4 Kalecki calcula que el ciclo de la inversión con perturbaciones incluidas abarca entre siete años y medio y ocho años, de ser prácticamente uno el coeficiente estático, el proceso se mantendría en tendencia y no crearía oscilaciones o tardarían mucho en producirse.

5 Para obtener estas ecuaciones sólo hay que aplicar diferencias logarítmicas en las variables de cuota salarial y nivel de empleo, y sustituir.

6 Para solucionar el sistema de ecuaciones (5.1) y (5.2) es necesario linearizar, pero Goodwin lo resuelve eliminando el factor tiempo e integrando, de donde obtiene la ecuación $(1 / \sigma) u+p v-(1 / \sigma-(\alpha+p)) \ln u-(\gamma+\alpha) \ln v$ igual a una constante definiendo $\theta_{1}=1 / \sigma, \eta_{1}=1 / \sigma-(\alpha+\beta), \theta_{2}=p, \eta_{2}=\gamma+\alpha$, sustituyendo en la ecuación anterior e integrando se puede transformar en $\Phi(u)=u_{i}^{\prime \prime} e^{-\theta, u}=v_{2}^{y} e^{-\theta_{1} v}=H \Psi(v)$.

7 Asimakopulos (1975, p. 330): «When it is no longer possible to increase output in the short period because of the absence of unemployed labour or unused productive capacity, the class struggle would be reflected (...) in the shares of total output accounted for by capitalists' expenditure. A stronger bargaining position of workers would be reflected in a diminution of capitalists' expenditure in real terms and thus in a higher share for workers in total income».

\section{BIBLIOGRAFÍA}

Asimakopulos, A. (1975). A Kaleckian theory of income distribution. Canadian Journal of Economics, 313-333.

Blecker, R. A. (2002). Distribution, demand and growth in neo-Kaleckian macro-models. In: Setterfield, M. (Ed.) The economics of demand-led growth. Cheltenham, UK: Edward Elgar.

Cárdenas, L. (2014). La teoría macroeconómica kaleckiana. Una visión general. XIV Jornadas de Economía Crítica. Valladolid.

Feiwel, G. R. (1981). Michal Kalecki: contribuciones a la teoría de la política económica. México DF, México: Fondo de Cultura Económica.

Goodwin, R. M. (1967). A growth cycle. In: Feinstein, C. H. (Ed.) Socialism, capitalism and economic growth. Cambridge University Press, 54-58.

Goodwin, R. M. (1982). Essays in economic dynamics. Macmillan Press.

Harvie, D. (2000). Testing Goodwin: growth cycles in ten OECD countries. Cambridge Journal of Economics, (24), 349-376.

Kaldor, N. (1955). Alternative theories of distribution. The Review of Economic Studies, (23), 83-100.

Kaldor, N. (1957). A model of economic growth. The economic journal, 591-624.

Kalecki, M. (1943). Political aspects of full employment. The Political Quarterly, (14), 322-330.

Kalecki, M. (1956). Teoría de la dinámica económica: Ensayo sobre los movimientos cíclicos ya largo plazo de la economía capitalista. México DF, México: Fondo de Cultura Económica.

Kalecki, M. (1968). Trend and Business Cycles Reconsidered. The Economic Journal, (78), 263-276.

Kalecki, M. (1971a). Class Struggle and the Distribution of National Income. Kyklos, (24), 1-9.

Kalecki, M. (1971b). Selected essays on the dynamics of the capitalist economy 1933-1970. Cambridge: Cambridge University Press.

Keynes, J. M. (1930). The Treatise on Money. Vol. 1. London, England: Macmillan.

Lavoie, M. (1995). The Kaleckian model of growth and distribution and its neo-Ricardian and neo-Marxian critiques. Cambridge Journal of Economics, (19), 789-818. 
Lavoie, M. (2014). Post-Keynesian Economics: New Foundations. UK: Edward Elgar Publishing. López, J., y Assous, M. (2010). Michal Kalecki. New York, EE. UU.: Palgrave Macmillan.

Palazuelos, E. y Fernández, R. (2007). La tasa de beneficio en la dinámica económica de los países europeos, 1984-2003. Estudios de Economía Aplicada, (25), 821-826.

Palley, T. I. (2014). A neo-Kaleckian-Goodwin model of capitalist economic growth monopoly power, managerial pay and labour market conflict. Cambridge Journal of Economics, 38(6), 1355-72.

Pasinetti, L. L. (1962). Rate of Profit and Income Distribution in Relation to the Rate of Economic Growth. The Review of Economic Studies, (29), 267-279.' 